\title{
Markers of coagulation dysfunction and inflammation in diabetic and non-diabetic COVID-19
}

\author{
Seshadri Reddy Varikasuvu ${ }^{1}$ [D $\cdot$ Saurabh Varshney ${ }^{2} \cdot$ Naveen Dutt $^{3}$
}

Published online: 5 September 2020

(c) Springer Science+Business Media, LLC, part of Springer Nature 2020

\begin{abstract}
Coagulation dysfunction and inflammatory status were compared between diabetic and non-diabetic COVID-19 patients. The standardized mean difference (SMD) and its 95\% confidence interval (CI) was computed for the difference of inflammatory and hypercoagulability markers. The levels of serum ferritin (standardized mean difference-SMD: 0.47, CI 0.17-0.77, $\mathrm{p}=0.002)$, C-reactive protein $(\mathrm{SMD}=0.53, \mathrm{CI} 0.20-0.86, \mathrm{p}=0.002)$, interleukin-6 $(\mathrm{SMD}=0.31, \mathrm{CI} 0.09-0.52, \mathrm{p}=0.005)$, fibrinogen ( $\mathrm{SMD}=0.31, \mathrm{CI} 0.09-0.54, \mathrm{p}=0.007)$ and $\mathrm{D}$-dimers $(\mathrm{SMD}=0.54, \mathrm{CI} 0.16-0.91, \mathrm{p}=0.005)$ were significantly higher in diabetic COVID-19 cases as compared to non-diabetic COVID-19 patients, suggesting more susceptibility of diabetic COVID-19 patients to coagulation dysfunction and inflammatory storm.
\end{abstract}

Keywords COVID-19 $\cdot$ Diabetes $\cdot$ D-dimer $\cdot$ Inflammation

\section{Highlights}

- The markers of coagulation dysfunction and inflammation were studied between diabetic and non-diabetic COVID-19 patients by meta-analysis.

- COVID-19 patients with diabetes have a significantly higher levels of coagulation dysfunction markers such as Fibrinogen $(\mathrm{SMD}=0.31, \mathrm{CI} 0.09-0.54, \mathrm{p}=0.007$ ) and D-dimers (SMD $=0.54$, CI 0.16-0.91, $\mathrm{p}=0.005$ ) than the non-diabetic COVID-19 cases.

- COVID-19 patients with diabetes have a significantly higher inflammatory markers such as C-reactive protein $(\mathrm{SMD}=0.53, \mathrm{CI} 0.20-0.86, \mathrm{p}=0.002)$, Interleukin-6 $(\mathrm{SMD}=0.31, \mathrm{CI} 0.09-0.52, \mathrm{p}=0.005)$ than the nondiabetic COVID-19 cases.

Seshadri Reddy Varikasuvu

lifeschemistry@live.com

1 Department of Biochemistry, All India Institute of Medical Sciences (AIIMS), Deoghar, Jharkhand 814152, India

2 All India Institute of Medical Sciences (AIIMS), Deoghar, Jharkhand, India

3 Department of Respiratory Medicine, All India Institute of Medical Sciences (AIIMS), Jodhpur, Rajasthan, India
- These results indicate that diabetic COVID-19 patients are more susceptibility to coagulation dysfunction and inlammatory storm.

\section{Introduction}

The world is struggling in lockdown for months since December of 2019 due to novel coronavirus disease (COVID-19) outbreak, a pandemic declared by the World Health Organization [1]. Research evidence is growing on the role of several symptoms, comorbidities, inflammation and hypercoagulability markers in relation to disease progression and deaths in COVID-19 patients. The incidence of diabetes, one of the leading causes of morbidity has been shown to be high and is associated with disease progression in COVID-19 [2,3].

Diabetic patients due to low pulmonary function have been reported to be more susceptible to intensive care admissions, mechanical ventilation and deaths due to COVID-19 than those without diabetes $[4,5]$. Though several studies have reported various inflammatory and coagulability markers such as serum ferritin, $\mathrm{C}$-reactive protein (CRP), interleukin-6 (IL-6), fibrinogen and D-dimers in relationship to disease severity and progression, much attention has to be paid to the comparisons between diabetic and non-diabetic COVID-19 cases [6-8]. 


\section{Methods}

In this pooled analysis, we aim to compare inflammatory storm and hypercoagulability status between diabetic and non-diabetic COVID-19 patients comprising a PROSPERO registered protocol (CRD42020186661). A total of 413 records were primarily identified. Of which, 39 relevant articles dealing with the inflammatory and hypercoagulation markers in COVID-19 patients were considered for full text evaluation. Out of these, 20 articles were excluded for not having relevant data, and 16 studies excluded for not comparing between diabetic and non-diabetic groups resulting in an inclusion of six observations from three studies for each of the variable between diabetic $(n=252)$ and non-diabetic $(n=497)$ COVID-19 cases [3-5]. These observations included for meta-analysis compared several markers between 252 diabetic and 497 non-diabetic cases. The study characteristics were presented in Table 1.

The standardized mean difference (SMD) with 95\% confidence intervals (CI) were obtained for the difference of inflammatory and hypercoagulability markers between diabetic and non-diabetic COVID-19 cases. The between study heterogeneity was examined by the Cochrane's $\mathrm{Q}$ statistic and expressed as the percentages of $\mathrm{I}^{2}$. A $p$ value of $<.05$ was considered statistically significant. A one-study leave-out sensitivity analysis was performed to validate the results. All analyses were conducted using Review Manager Version 5.3.

\section{Results}

The forest plots of this meta-analysis were shown in Fig. 1. With a significant between-study heterogeneity $\left(\mathrm{I}^{2}=64 \%\right.$, $\mathrm{p}<0.0001)$, the random-effects model showed significantly higher levels of inflammatory and hypercoagulability markers in diabetic COVID-19 group when compared to that of non-diabetic COVID-19 group (Fig. 1). The pooled SMD and 95\% CI were $0.43(0.30 ; 0.55)$. The overall effect size for SMD calculated as $\mathrm{Z}$ was 6.67 $(\mathrm{p}<0.0001)$. The sub-group analysis showed that serum ferritin (SMD: $0.4795 \%$ CI 0.17-0.77, $\mathrm{p}=0.002$ ), CRP (SMD: 0.53 95\% CI 0.20-0.86, $\mathrm{p}=0.002$ ), IL-6 (SMD: $0.3195 \%$ CI $0.09-0.52, \mathrm{p}=0.005$ ), fibrinogen (SMD: $0.3195 \%$ CI $0.09-0.54, \mathrm{p}=0.007$ ), and D-dimer (SMD: $0.5495 \%$ CI $0.16-0.91, \mathrm{p}=0.005$ ) levels are significantly elevated in diabetic patients as compared to non-diabetic counterparts with COVID-19. The sensitivity analysis showed that no single study had significantly influenced the overall result, which remained to be stable and significant after leaving-out any particular study/observation.

\section{Discussion}

These results show that the inflammatory and hypercoagulability markers significantly increase in diabetic group of COVID-19 patients when compared to their non-diabetic counterparts. Various reports suggest that diabetes activate several pathways leading to T-cell differentiation, immune system imbalance, pro- and anti-inflammation imbalance [4, 9]. Diabetes has been reported to be associated with infection and disease progression $[3,10]$. According to recent research, virus invasion results in induction of coagulation activation, inflammatory responses, hypercoagulability induction and cytokine storms which may eventually cause disease progression in COVID-19 patients [2, 3].

The significant rise in ferritin, CRP and IL-6 levels reflect monocyte-macrophage activation resulting in inflammatory storm and cytokine storm. With its expression time longer than others, the cytokine IL-6 levels have been reported to be better predictors of disease progression [11]. It is known that during inflammatory storm, as a result of plasmin activation, the significant rise in D-dimer level indicates hypercoagulability $[5,7]$. The significant rise in fibrinogen and D-dimer indicate diabetic COVID-19 patients are more susceptible to hypercoagulable state/intravascular coagulation. It is noteworthy that the association of diabetes and hyperglycemia with disease progression has been linked to increased inflammation, hypercoagulability and lung dysfunction in COVID-19 [3, 12].

It is well documented in several studies $[6,13,14]$ that inflammatory and hypercoagulation status increase in COVID-19 cases as compared to non-COVID-19 respiratory illness. And, the presence of diabetes could further influence the magnitude of inflammatory and coagulation dysfunction in COVID-19. Strikingly, a recent study showed a significant increase in these markers in diabetic group as compared to non-diabetic group of COVID-19 patients without other comorbidities, indicating the independent impact of diabetes [3]. Moreover, the presence of diabetes has been associated with the poorer survival of COVID-19 cases with a hazard ratio (HR) of 3.17 (95\% CI 1.93-5.20). And, this association remained to be significant even after adjusting for age and other comorbidities like hypertension, cardiovascular and cerebrovascular diseases $(\mathrm{HR}=1.53,95 \% \mathrm{CI}$ 1.02-2.30). In a study by Zhang et al. [5], after adjusting for 
Table 1 The characteristics of included studies

\begin{tabular}{|c|c|c|c|c|c|c|c|c|c|c|c|}
\hline \multirow[t]{2}{*}{ Characteristic } & & \multicolumn{10}{|c|}{ Study } \\
\hline & & \multicolumn{3}{|c|}{ Guo et al. [3] } & \multicolumn{4}{|c|}{ Yan et al. [4] } & \multicolumn{3}{|c|}{ Zhang et al. [5] } \\
\hline \multicolumn{2}{|l|}{ Country } & \multicolumn{3}{|c|}{ China } & \multicolumn{4}{|c|}{ China } & \multicolumn{3}{|c|}{ China } \\
\hline \multicolumn{2}{|l|}{ Study type } & \multicolumn{3}{|c|}{ Retrospective observational study } & \multicolumn{4}{|c|}{ Retrospective observational study } & \multicolumn{3}{|c|}{ Retrospective observational study } \\
\hline \multicolumn{2}{|l|}{ Criteria } & \multicolumn{3}{|c|}{ WHO interim guidance } & \multicolumn{4}{|c|}{ WHO interim guidance } & \multicolumn{3}{|c|}{$\begin{array}{l}\text { Chinese National Health } \\
\text { Committee (version 7) }\end{array}$} \\
\hline RT-PCR & & \multicolumn{3}{|c|}{ Yes } & \multicolumn{4}{|l|}{ Yes } & \multicolumn{3}{|l|}{ Yes } \\
\hline Outcomes & & \multicolumn{3}{|c|}{$\begin{array}{l}\text { Comparisons between diabetic and non- } \\
\text { diabetic cases }\end{array}$} & \multicolumn{4}{|c|}{$\begin{array}{l}\text { Comparisons between diabetic and non- } \\
\text { diabetic cases }\end{array}$} & \multicolumn{3}{|c|}{$\begin{array}{l}\text { Comparisons between diabetic } \\
\text { and non-diabetic cases }\end{array}$} \\
\hline \multirow{3}{*}{$\begin{array}{l}\text { Overall and } \\
\text { between } \\
\text { group com- } \\
\text { parisons }\end{array}$} & \multicolumn{11}{|l|}{ Study } \\
\hline & \multicolumn{3}{|c|}{ Guo et al. [3] } & \multicolumn{3}{|c|}{ Yan et al. [4] } & \multicolumn{5}{|c|}{ Zhang et al. [5] } \\
\hline & $\begin{array}{l}\text { Overall } \\
\text { COVID-19 }\end{array}$ & $\begin{array}{l}\text { Diabetic vs. } \\
\text { non-Dia- } \\
\text { betic }\end{array}$ & $\begin{array}{l}\text { Diabetic vs. } \\
\text { non-diabetic } \\
\text { (No-CUD) }\end{array}$ & $\begin{array}{l}\text { Overa } \\
\text { COVl }\end{array}$ & -19 & $\begin{array}{l}\text { Diabetic vs. } \\
\text { non-diabetic }\end{array}$ & $\begin{array}{l}\text { Overall } \\
\text { COVID-19 }\end{array}$ & $\begin{array}{l}\text { Diabe } \\
\text { non-d }\end{array}$ & $\begin{array}{l}\text { ic vs. } \\
\text { abetic }\end{array}$ & $\begin{array}{l}\text { Diabetic vs. } \\
\text { hyper- } \\
\text { glycemia } \\
\text { with other } \\
\text { comorbidi- } \\
\text { ties }\end{array}$ & $\begin{array}{l}\text { Hyperglyce- } \\
\text { mia vs. non- } \\
\text { diabetic }\end{array}$ \\
\hline Total (n) & 174 & 37 vs. 137 & 24 vs. 26 & $\begin{array}{r}193 \text { w } \\
\text { seve } \\
\text { CO }\end{array}$ & & 48 vs. 145 & 166 & $61 \mathrm{vs}$ & & 61 vs. 21 & 21 vs 84 \\
\hline Age & 59 & 61 vs. 58 & 61 vs. 32 & 64 & & 70 vs. 60 & 62.7 & 65.6 & s. 59.4 & 65.6 vs. 67.6 & 67.6 vs. 59.4 \\
\hline Male (n) & 76 & 20 vs. 56 & 12 vs. 9 & 114 & & 33 vs. 81 & 85 & 33 vs & & 33 vs. 11 & 11 vs. 41 \\
\hline Female (n) & 98 & 17 vs. 81 & 12 vs. 17 & 79 & & 15 vs. 64 & 81 & $28 \mathrm{vs}$ & & 28 vs. 10 & 10 vs. 43 \\
\hline \multicolumn{12}{|c|}{ Signs and symptoms (n) } \\
\hline $\begin{array}{l}\text { Fever/ } \\
\text { fatigue/ } \\
\text { headache }\end{array}$ & $136 / 47 / 12$ & $\begin{array}{l}22 \text { vs. } \\
114 / 11 \text { vs. } \\
36 / 2 \text { vs. } 10\end{array}$ & $\begin{array}{l}18 \text { vs. } 22 / 5 \\
\text { vs. } 9 / 1 \\
\text { vs. } 3\end{array}$ & $173 / 1$ & $1 / 21$ & $\begin{array}{l}43 \text { vs. } \\
\text { 130/28 vs. } \\
73 / 5 \text { vs. } 16\end{array}$ & $139 / 99 / 53$ & $\begin{array}{c}53 \text { vs. } \\
\text { vs. } \\
\text { vs. }\end{array}$ & $\begin{array}{l}70 / 35 \\
9 / 17 \\
7\end{array}$ & $\begin{array}{l}53 \text { vs. } 46 / 35 \\
\text { vs. } 15 / 17 \\
\text { vs. } 9\end{array}$ & $\begin{array}{l}16 \text { vs. } 70 / 15 \\
\text { vs. } 49 / 9 \\
\text { vs. } 27\end{array}$ \\
\hline $\begin{array}{l}\text { Chill/ } \\
\text { cough/ } \\
\text { dizziness }\end{array}$ & $119 / 56 / 23$ & $\begin{array}{l}21 \text { vs. } 98 / 8 \\
\text { vs. } 48 / 6 \\
\text { vs. } 17\end{array}$ & $\begin{array}{l}19 \text { vs. } \\
\text { 20/11vs. } \\
\text { 15/4 vs. } 2\end{array}$ & NA/1 & $5 / \mathrm{NA}$ & $\begin{array}{l}\text { NA/37 vs. } \\
\text { 98/NA }\end{array}$ & NA/136/NA & $\begin{array}{r}\mathrm{NA} / 5 \\
71 / \mathrm{I}\end{array}$ & & $\begin{array}{l}\text { NA/50 vs. } \\
15 / \mathrm{NA}\end{array}$ & $\begin{array}{l}\text { NA/15 vs. } \\
\text { 71/NA }\end{array}$ \\
\hline $\begin{array}{l}\text { Chest pain/ } \\
\text { chest } \\
\text { tightness/ } \\
\text { shortness } \\
\text { of breath }\end{array}$ & $15 / 45 / 42$ & $\begin{array}{c}1 \text { vs. } 14 / 5 \\
\text { vs. } 40 / 5 \\
\text { vs. } 37\end{array}$ & $\begin{array}{l}0 \text { vs. } 1 / 2 \text { vs. } \\
4 / 5 \text { vs. } 4\end{array}$ & $10 / \mathrm{N}$ & $/ 115$ & $\begin{array}{l}1 \text { vs. 9/ } \\
\text { NA/33 vs. } \\
82\end{array}$ & 25/NA/115 & $\begin{array}{c}11 \mathrm{vs} \\
\mathrm{NA} \\
55\end{array}$ & $\begin{array}{l}10 / \\
44 \text { vs. }\end{array}$ & $\begin{array}{l}11 \text { vs. } 4 / \\
\text { NA/44 vs. } \\
16\end{array}$ & $\begin{array}{l}4 \text { vs. } 10 / \\
\text { NA/16 vs. } \\
55\end{array}$ \\
\hline $\begin{array}{c}\text { Myalgia/ } \\
\text { pharyn- } \\
\text { galgia/ } \\
\text { nausea- } \\
\text { vomiting }\end{array}$ & $36 / 9 / 17$ & $\begin{array}{l}6 \text { vs. } 30 / 1 \\
\text { vs. } 8 / 5 \text { vs. } \\
12\end{array}$ & $\begin{array}{l}3 \text { vs. } 4 / 0 \text { vs. } \\
4 / 4 \text { vs. } 0\end{array}$ & $\mathrm{NA} / \mathrm{N}$ & / $/ 19$ & $\begin{array}{l}\text { NA/NA/4 } \\
\text { vs. } 15\end{array}$ & NA/NA/73 & $\begin{array}{l}\mathrm{NA} / \mathrm{N} \\
\text { vs. }\end{array}$ & $\begin{array}{l}\mathrm{A} / 29 \\
4\end{array}$ & $\begin{array}{l}\text { NA/NA/29 } \\
\text { vs. } 11\end{array}$ & $\begin{array}{l}\text { NA/NA/11 } \\
\text { vs. } 34\end{array}$ \\
\hline $\begin{array}{l}\text { Anorexia/ } \\
\text { diarrhoea }\end{array}$ & $\mathrm{NA} / 21$ & NA/3 vs. 18 & NA/3 vs. 4 & $68 / 51$ & & $\begin{array}{l}21 \text { vs. } 47 / 10 \\
\text { vs. } 41\end{array}$ & $75 / 77$ & $\begin{array}{c}27 \mathrm{vs} \\
\text { vs. }\end{array}$ & $\begin{array}{l}38 / 30 \\
7\end{array}$ & $\begin{array}{l}27 \text { vs. } 10 / 30 \\
\text { vs. } 10\end{array}$ & $\begin{array}{l}10 \text { vs. } 38 / 10 \\
\text { vs. } 37\end{array}$ \\
\hline $\begin{array}{l}\text { Comorbidi- } \\
\text { ties (n) }\end{array}$ & NA & NA & NA & 94 & & 29 vs. 65 & NA & NA & & NA & NA \\
\hline $\begin{array}{l}\text { Hyperten- } \\
\text { sion/ } \\
\text { cardio- } \\
\text { vascular } \\
\text { disease/ } \\
\text { malig- } \\
\text { nancy }\end{array}$ & $43 / 32 / 17$ & $\begin{array}{c}10 \text { vs. } 33 / 12 \\
\text { vs. } 20 / 1 \\
\text { vs. } 16\end{array}$ & None & $73 / 31$ & & $\begin{array}{c}24 \text { vs. } 49 / 13 \\
\text { vs. } 18 / \mathrm{NA}\end{array}$ & $76 / 30 / 3$ & $\begin{array}{c}35 \text { vs } \\
\text { vs. } \\
\text { vs. }\end{array}$ & $\begin{array}{l}30 / 16 \\
0 / 3\end{array}$ & $\begin{array}{l}35 \text { vs. } 11 / 16 \\
\text { vs. } 4 / 3 \\
\text { vs. } 0\end{array}$ & $\begin{array}{l}11 \text { vs. } 30 / 4 \\
\text { vs. } 10 / 0 \\
\text { vs. } 0\end{array}$ \\
\hline
\end{tabular}


Table 1 (continued)

\begin{tabular}{|c|c|c|c|c|c|c|c|c|c|}
\hline \multirow{3}{*}{$\begin{array}{l}\text { Overall and } \\
\text { between } \\
\text { group com- } \\
\text { parisons }\end{array}$} & \multicolumn{9}{|l|}{ Study } \\
\hline & \multicolumn{3}{|l|}{ Guo et al. [3] } & \multicolumn{2}{|l|}{ Yan et al. [4] } & \multicolumn{4}{|c|}{ Zhang et al. [5] } \\
\hline & $\begin{array}{l}\text { Overall } \\
\text { COVID-19 }\end{array}$ & $\begin{array}{l}\text { Diabetic vs. } \\
\text { non-Dia- } \\
\text { betic }\end{array}$ & $\begin{array}{l}\text { Diabetic vs. } \\
\text { non-diabetic } \\
\text { (No-CUD) }\end{array}$ & $\begin{array}{l}\text { Overall } \\
\text { COVID-19 }\end{array}$ & $\begin{array}{l}\text { Diabetic vs. } \\
\text { non-diabetic }\end{array}$ & $\begin{array}{l}\text { Overall } \\
\text { COVID-19 }\end{array}$ & $\begin{array}{l}\text { Diabetic vs. } \\
\text { non-diabetic }\end{array}$ & $\begin{array}{l}\text { Diabetic vs. } \\
\text { hyper- } \\
\text { glycemia } \\
\text { with other } \\
\text { comorbidi- } \\
\text { ties }\end{array}$ & $\begin{array}{l}\text { Hyperglyce- } \\
\text { mia vs. non- } \\
\text { diabetic }\end{array}$ \\
\hline $\begin{array}{l}\text { Pulmonary } \\
\text { disease/ } \\
\text { kidney } \\
\text { disease/ } \\
\text { liver } \\
\text { disease }\end{array}$ & $14 / 13 / 8$ & $\begin{array}{l}2 \text { vs. } 12 / 1 \\
\text { vs. } 12 / 0 \\
\text { vs. } 8\end{array}$ & None & $14 / 4 / 1$ & $\begin{array}{l}4 \text { vs. } 10 / 0 \\
\text { vs. } 4 / 0 \\
\text { vs. } 1\end{array}$ & 19/9/NA & $\begin{array}{l}9 \text { vs. } 9 / 3 \text { vs. } \\
\text { 6/NA }\end{array}$ & $\begin{array}{l}9 \text { vs. } 1 / 3 \text { vs. } \\
0 / \mathrm{NA}\end{array}$ & $\begin{array}{l}1 \text { vs. } 9 / 0 \text { vs. } \\
\text { 6/NA }\end{array}$ \\
\hline $\begin{array}{l}\text { Immune } \\
\text { defi- } \\
\text { ciency/ } \\
\text { Hepa- } \\
\text { titis B/ } \\
\text { cerebro- } \\
\text { vascular } \\
\text { disease }\end{array}$ & $4 / 2 / 13$ & $\begin{array}{l}0 \text { vs. } 4 / 0 \text { vs. } \\
2 / 1 \text { vs. } 12\end{array}$ & None & NA/NA/8 & $\begin{array}{l}\text { NA/NA/5 } \\
\text { vs. } 3\end{array}$ & NA/NA/12 & $\begin{array}{l}\text { NA/NA/6 } \\
\text { vs. } 3\end{array}$ & $\begin{array}{l}\text { NA/NA/6 } \\
\text { vs. } 3\end{array}$ & $\begin{array}{l}\text { NA/NA/3 } \\
\text { vs. } 3\end{array}$ \\
\hline $\begin{array}{l}\text { Thyroid } \\
\text { disease/ } \\
\text { digestive } \\
\text { system } \\
\text { disorders/ } \\
\text { others }\end{array}$ & NA/NA/NA & NA/NA/NA & NA/NA/NA & NA/NA/NA & NA/NA/NA & $3 / 5 / 91$ & $\begin{array}{l}1 \text { vs. } 2 / 2 \text { vs. } \\
2 / 37 \text { vs. } 43\end{array}$ & $\begin{array}{l}1 \text { vs. } 0 / 2 \text { vs. } \\
1 / 37 \text { vs. } \\
11\end{array}$ & $\begin{array}{l}0 \text { vs. } 2 / 1 \text { vs. } \\
2 / 11 \text { vs. } 43\end{array}$ \\
\hline $\begin{array}{l}\text { Mortalities } \\
\text { (n) }\end{array}$ & 9 & 4 vs. 5 & 4 vs. 0 & 108 & 39 vs. 69 & 24 & 13 vs. 8 & 13 vs. 3 & 3 vs. 8 \\
\hline
\end{tabular}

NA not available, No-CUD no other comorbidities

confounders like; age, sex, BMI and other comorbidities, a significantly higher rate of composite outcomes (ICU admission/mechanical ventilation/deaths) in both hyperglycemia (odds ratio-OR $=5.47,95 \%$ CI 1.51-19.82) and diabetic groups $(\mathrm{OR}=2.61,95 \% \mathrm{CI} 0.86-7.88)$ than the non-diabetic COVID-19 group were reported.

Our pooled analysis shows that diabetic COVID-19 patients are more susceptible to coagulation dysfunction and inflammation than the non-diabetic COVID-19 cases.
The sensitivity analysis indicated the robustness of overall result. Though, the included studies matched the diabetic and non-diabetic groups for overall comorbidities [4] and all comorbidities except for CVD [3] and hypertension [5], the results should be interpreted with a caution that diabetes may coexist with other conditions in COVID-19 patients. Therefore, further well controlled studies are needed in future to establish an independent role of diabetes in COVID-19. 


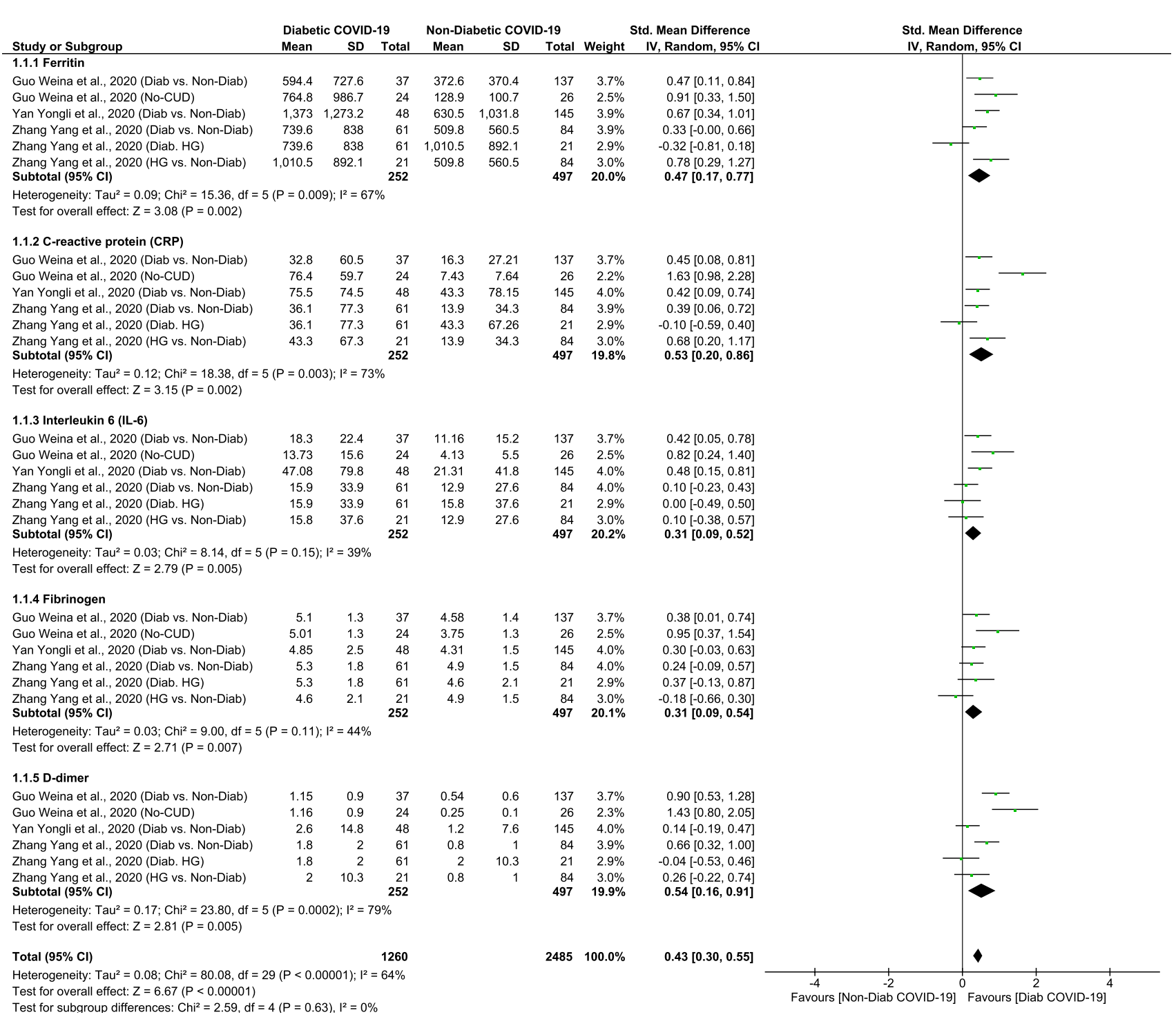

Fig. 1 The forest plots comparing hypercoagulability and inflammatory status between diabetic and non-diabetic COVID-19 cases

Acknowledgements The authors are is very much thankful to Prof. TiejunTong and his PhD Scholars (Luo Dehui and Jindong Shi), HongKong Baptist University for their statistical expertise and help in the methodology.Dr. S.R. Varikasuvu specially acknowledges Varikasuvu Bhairavi Sisters (Sahasra \& Aagneya) for the time I could not give you during this work.

\section{Compliance with ethical standards}

Conflict of interest All authors declare that they have no conflict of interest.

\section{References}

1. Cucinotta D, Vanelli M (2020) WHO declares COVID-19 a pandemic. Acta Biomed 91:157-160
2. Li J, Wang X, Chen J et al (2020) COVID-19 infection may cause ketosis and ketoacidosis. Diabetes Obes Metab. https://doi. org/10.1111/dom.14057

3. Guo W, Li M, Dong Y et al (2020) Diabetes is a risk factor for the progression and prognosis of COVID-19. Diabetes Metab Res Rev. https://doi.org/10.1002/dmrr.3319

4. Yan Y, Yang Y, Wang F et al (2020) Clinical characteristics and outcomes of patients with severe covid-19 with diabetes. BMJ Open Diabetes Res Care 8:e001343. https://doi.org/10.1136/bmjdr c-2020-001343

5. Zhang Y, Li H, Zhang J et al (2020) The clinical characteristics and outcomes of diabetes mellitus and secondary hyperglycaemia patients with coronavirus disease 2019: a single-center, retrospective, observational study in Wuhan. Diabetes Obes Metab 22:1443-1454. https://doi.org/10.1111/dom.14086

6. Spiezia L, Boscolo A, Poletto F et al (2020) COVID-19-related severe hypercoagulability in patients admitted to intensive care unit for acute respiratory failure. Thromb Haemost 120:998-1000. https://doi.org/10.1055/s-0040-1710018 
7. Li Y, Zhao K, Wei H et al (2020) Dynamic relationship between D-dimer and COVID-19 severity. Br J Haematol 190:e24-e27. https://doi.org/10.1111/bjh.16811

8. Connors JM, Levy JH (2020) COVID-19 and its implications for thrombosis and anticoagulation. Blood 135:2033-2040. https:// doi.org/10.1182/blood.2020006000

9. Jagannathan-Bogdan M, McDonnell ME, Shin H et al (2011) Elevated proinflammatory cytokine production by a skewed $\mathrm{T}$ cell compartment requires monocytes and promotes inflammation in type 2 diabetes. J Immunol 186:1162-1172

10. Yang JK, Feng Y, Yuan MY et al (2006) Plasma glucose levels and diabetes are independent predictors for mortality and morbidity in patients with SARS. Diabetic Med 23:623-628

11. Tanaka T, Narazaki M, Kishimoto T (2016) Immunotherapeutic implications of IL-6 blockade for cytokine storm. Immunotherapy 8:959-970
12. Sardu C, D'Onofrio N, Balestrieri ML et al (2020) Outcomes in patients with hyperglycemia affected by COVID-19: can we do more on glycemic control? Diabetes Care 43:1408-1415. https:// doi.org/10.2337/dc20-0723

13. Di Micco P, Russo V, Carannante $\mathrm{N}$ et al (2020) Clotting factors in COVID-19: epidemiological association and prognostic values in different clinical presentations in an Italian Cohort. J Clin Med 9:1371. https://doi.org/10.3390/jcm9051371

14. Han H, Yang L, Liu R et al (2020) Prominent changes in blood coagulation of patients with SARS-CoV-2 infection. Clin Chem Lab Med 58:1116-1120. https://doi.org/10.1515/cclm-2020-0188

Publisher's Note Springer Nature remains neutral with regard to jurisdictional claims in published maps and institutional affiliations. 